\title{
Grade classification of neuroepithelial tumors using high- resolution magic-angle spinning proton nuclear magnetic resonance spectroscopy and pattern recognition
}

\author{
CHEN WenXue ${ }^{1,2^{*}}$, LOU HaiYan ${ }^{3,4}$, ZHANG HongPing $^{5}$, NIE Xiu ${ }^{6}$, LAN WenXian $^{1}$, \\ YANG YongXia ${ }^{1}$, XIANG Yun ${ }^{1}$, QI JianPin ${ }^{3}$, LEI Hao ${ }^{1}$, TANG HuiRu ${ }^{1}$, \\ CHEN FenEr ${ }^{2} \&$ DENG Feng ${ }^{1}$ \\ ${ }^{1}$ State Key Laboratory of Magnetic Resonance and Atomic and Molecular Physics, Wuhan Institute of Physics and Mathematics, \\ Chinese Academy of Sciences, Wuhan 430071, China; \\ ${ }^{2}$ Fudan-DSM Joint Laboratory, Department of Chemistry, Fudan University, Shanghai 200433, China; \\ ${ }^{3}$ Tongji Hospital, Tongji Medical College, Huazhong University of Science and Technology, Wuhan 430030, China; \\ ${ }^{4}$ The first Affiliated Hospital, College of Medicine, Zhejiang University, Hangzhou 310003, China; \\ ${ }^{5}$ Zhongnan Hospital, Medical College, Wuhan University, Wuhan 430071, China; \\ ${ }^{6}$ Union Hospital, Tongji Medical College, Huazhong University of Science and Technology, Wuhan 430022, China
}

Received December 17, 2010; accepted June 20, 2011

\begin{abstract}
Clinical data have shown that survival rates vary considerably among brain tumor patients, according to the type and grade of the tumor. Metabolite profiles of intact tumor tissues measured with high-resolution magic-angle spinning proton nuclear magnetic resonance spectroscopy (HRMAS ${ }^{1} \mathrm{H}$ NMRS) can provide important information on tumor biology and metabolism. These metabolic fingerprints can then be used for tumor classification and grading, with great potential value for tumor diagnosis. We studied the metabolic characteristics of 30 neuroepithelial tumor biopsies, including two astrocytomas (grade I), 12 astrocytomas (grade II), eight anaplastic astrocytomas (grade III), three glioblastomas (grade IV) and five medulloblastomas (grade IV) from 30 patients using HRMAS ${ }^{1} \mathrm{H}$ NMRS. The results were correlated with pathological features using multivariate data analysis, including principal component analysis (PCA). There were significant differences in the levels of $\mathrm{N}$-acetyl-aspartate (NAA), creatine, myo-inositol, glycine and lactate between tumors of different grades $(P<0.05)$. There were also significant differences in the ratios of $\mathrm{NAA} / \mathrm{creatine}$, lactate/creatine, $m y o$-inositol/creatine, glycine/creatine, scyllo-inositol/creatine and alanine/creatine $(P<0.05)$. A soft independent modeling of class analogy model produced a predictive accuracy of $87 \%$ for high-grade (grade III-IV) brain tumors with a sensitivity of $87 \%$ and a specificity of $93 \%$. HRMAS ${ }^{1} \mathrm{H}$ NMR spectroscopy in conjunction with pattern recognition thus provides a potentially useful tool for the rapid and accurate classification of human brain tumor grades.
\end{abstract}

neuroepithelial tumor, grade classification, high-resolution magic-angle spinning nuclear magnetic resonance (HRMAS NMR) spectroscopy, metabonomics, pattern recognition

Citation: Chen W X, Lou H Y, Zhang H P, et al. Grade classification of neuroepithelial tumors using high-resolution magic-angle spinning proton nuclear magnetic resonance spectroscopy and pattern recognition. Sci China Life Sci, 2011, 54: 606-616, doi: 10.1007/s11427-011-4193-7

Brain tumors are associated with poor survival and high recurrence rates; less than $30 \%$ of adult patients survive for

*Corresponding author (email: wxchen@fudan.edu.cn) five years after diagnosis of a primary malignant brain tumor [1-4]. Gliomas such as astrocytomas and glioblastomas account for about $45 \%$ of all primary human brain tumors seen in the clinic, with incidences of around 5-10 per 
100000 of the general population [5]. Early detection and accurate diagnosis are therefore vitally important for their appropriate treatment. The clinical diagnosis of brain tumors currently relies almost exclusively on the pathological evaluation of morphological changes in tissue specimens. However, the diagnosis of many malignant tumors, especially neuroepithelial tumors (e.g., oligodendroglioma, medulloblastoma), remains a challenge for clinicians and neuropathologists [6-8].

Modern diagnostic techniques such as X-ray computed tomography [9], magnetic resonance imaging [10,11], and positron-emission tomography $[12,13]$ form the bases for patient treatment plans, and have all been used clinically to characterize human brain tumors. However, the techniques are often used to identify and assess tumor size, and directly detect tumor site and location, and thus remain complementary to histopathology in terms of clinical diagnosis.

In vivo ${ }^{1} \mathrm{H}$ magnetic resonance spectroscopy (MRS) [14-17] and in vitro ${ }^{1} \mathrm{H}$ nuclear magnetic resonance (NMR) spectroscopy of tissue extracts [18-21] have provided more information on metabolites correlated with the histopathology of tissue specimens, and have helped to improve our understanding of tumor biology, with benefits in terms of the diagnostic accuracy of human brain tumors. However, although in vivo ${ }^{1} \mathrm{H}$ MRS [14-17] is the only non-invasive in vivo metabolite-detection technique, its limited spectral resolution and detection sensitivity means that it can often only detect a few metabolites, such as lipids (Lip), total creatine (tCre), total choline (tCho), lactate (Lac), glutamate (Glu) (or glutamine, Gln), and N-acetyl-aspartate (NAA). Furthermore, the spectral baseline and signal-to-noise ratio may be inadequate for quantitative analysis. By contrast, although in vitro ${ }^{1} \mathrm{H}$ NMR spectroscopy of tissue extracts [18-21] has a much higher resolution, its use remains questionable: (i) The extraction method requires large amounts of tissue (at least $50 \mathrm{mg}$ ); (ii) the destructive nature of the extraction may alter the corresponding spectrum to an unknown degree, although the length of the extraction procedure is acceptable ( $>24 \mathrm{~h}$ ); (iii) the extraction method usually discriminates between metabolites on the basis of solubility in a particular solvent, and prevents the direct observation of semi-mobile membrane lipids. Thus, complementary methods are needed for the rapid and direct extraction of further information on the metabolic characteristics of tumors tissues.

High-resolution magic-angle spinning (HRMAS) ${ }^{1} \mathrm{H}$ NMR spectroscopy of intact biological tissues has recently proven to be a useful technique for the rapid classification and grading diagnosis of tumors [22-25]. This technique only requires minimal sample preparation (about $5 \mathrm{~min}$ ), and allows the observation of most of the tissue metabolites and their dynamic interactions in small sample (around $10 \mathrm{mg}$ ). Furthermore, the shorter acquisition time $(<20 \mathrm{~min})$ of the procedure considerably reduces the risk of chemical alterations of the biopsy and prevents extensive tissue degradation [26]. When used in conjunction with multivariate data analy- sis (MVDA)-based pattern recognition (PR) methods, such as principal component analysis (PCA), semi-automated NMR methods have become an important part of the ex vivo tissue metabonomics platform, and currently provides the only known non-invasive tissue metabonomics technique. These advantages are already being exploited $[27,28]$ to provide a convenient, rapid and accurate complementary method to biochemical studies for the diagnosis and classification of human tumors.

In this study, we used HRMAS ${ }^{1} \mathrm{H}$ NMRS methods in conjunction with MVDA to analyze the metabonomic characteristics of 30 human neuroepithelial tumors with the aims of further understanding the differences between various grades of tumors at the metabolic level, and exploring the feasibility of a new grade-classification technique to complement conventional techniques.

\section{Materials and methods}

\subsection{Collection and pathological assessment of brain tissue samples}

Two astrocytoma (grade I), 12 astrocytoma (grade II), eight anaplastic astrocytoma (grade III), three glioblastoma (grade IV) and five medulloblastoma (grade IV) specimens were obtained from 30 patients at the Department of Neurosurgery, Tongji Hospital, Tongji College of Medicine, Huazhong University of Science and Technology, and Zhongnan Hospital, Medical College of Wuhan University. Each specimen was divided into two parts after craniotomy ( $<30 \mathrm{~min}$ of tissue collection). One part was fixed in $10 \%$ neutral buffered formalin solution and embedded in paraffin, and used for routine pathological examination. Tissue sections $(5 \mu \mathrm{m}$ thick) were stained with hematoxylin and eosin (H\&E) for microscopic examination, and characterized according to the 2000 revised World Health Organization criteria for brain tumors. The other part to be used for MAS was immediately snap-frozen in liquid nitrogen and subsequently stored at $-80^{\circ} \mathrm{C}$ until HRMAS ${ }^{1} \mathrm{H}$ NMRS measurement. This study was reviewed and approved by the local ethics committee and informed consent for the use of their tissue samples for research purposes was obtained from all patients.

All brain tumor samples were classified into five groups, based on type and grade. Patient data are summarized in Table 1 . The patients had a wide age range (10-71 years), with a higher percentage of males $(n=20)$ than females $(n=10)$. However, based on the limited number of cases, no obvious age-related trends were detectable, except for the observation that medulloblastomas occurred more frequently in childhood and adolescence.

\subsection{HRMAS ${ }^{1} \mathrm{H}$ NMR spectroscopy of brain tumor tissues}

Each individual tumor sample $(20-30 \mathrm{mg})$ was rinsed in 
Table 1 Histopathological data for 30 brain tumor specimens from 30 patients

\begin{tabular}{|c|c|c|c|c|c|c|}
\hline \multirow{2}{*}{ Group (number) } & \multirow{2}{*}{ Pathological diagnosis } & \multirow{2}{*}{ Grade (I-IV) } & \multicolumn{2}{|c|}{ Gender } & \multicolumn{2}{|c|}{ Age of patient (year) } \\
\hline & & & Male & Female & Range & Mean \pm SD \\
\hline Group $1(n=2)$ & astrocytoma & I & 1 & 1 & $36-63$ & $50 \pm 19.1$ \\
\hline Group $2(n=12)$ & astrocytoma & II & 8 & 4 & $21-63$ & $38 \pm 12.6$ \\
\hline Group 3 (n=8) & astrocytoma & III & 3 & 5 & $36-71$ & $49 \pm 11.7$ \\
\hline Group $4(n=3)$ & glioblastoma & IV & 3 & 0 & $22-41$ & $32 \pm 9.5$ \\
\hline Group $5(n=5)$ & medulloblastoma & IV & 5 & 0 & $10-32$ & $19 \pm 9.4$ \\
\hline
\end{tabular}

$\mathrm{D}_{2} \mathrm{O}$ saline at low temperature $\left(4^{\circ} \mathrm{C}\right)$, and placed into a 4-mm quartz rotor, especially designed for HRMAS spectroscopy. The total preparation time for each sample was less than $5 \mathrm{~min}$. All HRMAS ${ }^{1} \mathrm{H}$ NMR spectra were acquired at $298 \mathrm{~K}$ using the methanol method [29] to calibrate the temperature of the HRMAS probe on a Varian INVOA $599.906 \mathrm{MHz}$ spectrometer (California, USA) equipped with a standard gHX Nanoprobe (California, USA) at a spin rate of $2 \mathrm{kHz}$. Two ${ }^{1} \mathrm{H}$ NMR spectra for each sample were recorded using one-dimensional single-pulse sequence and standard Carr-Purcell-Meiboom-Gill (CPMG) spin-echo pulse sequence [RD- $90^{\circ}-\left(\tau-180^{\circ}-\tau\right) n$-ACQ] [26,30]. Water signals were pre-saturated in both cases by weak continuous wave irradiation on water resonance during the recycle delay (RD). The $90^{\circ}$ pulse length was adjusted to $4.2-5.7 \mu \mathrm{s}$ for each individual sample, and 128 scans were collected using $16 \mathrm{k}$ data, a recycle delay of $2 \mathrm{~s}$ and a spectral width of $12 \mathrm{kHz}$ for all samples. In the case of the CPMG experiments, a total spin-spin relaxation delay $(2 n \tau)(\tau=(240-250)$ $\mu \mathrm{s})$ of $32 \mathrm{~ms}$ was used for all samples. The total acquisition time for each sample was less than $20 \mathrm{~min}$.

The free induction decays were multiplied by an exponential window function with a $1.0-\mathrm{Hz}$ line-broadening factor prior to Fourier transformation. All sample spectra were manually phase- and baseline-corrected using XWINNMR 2.0 software (Bruker Analytik, Germany) after converting the data into the correct format (whole spectrum). The proton chemical shifts were referenced internally to the centre of the lactate methyl doublet at $1.33 \mathrm{ppm}$.

When more cases are studied, simple comparisons between spectra are inadequate for obtaining all the useful information on tissue metabolite changes because of the spectral complexity and the large quantity of data. The most effective method is thus to provide an average spectrum for each group of tumors. The average spectrum for each group can be obtained as follows: The spectral region $(\delta 0.50-4.50)$ is divided into 4000 buckets with a uniform width of 0.001 $\mathrm{ppm}$. The integral of each bucket is then normalized to the total integrals over the entire region $(\delta 0.50-4.50)$. These normalized spectra for all sub-groups of tumors are averaged numerically.

\subsection{Data reduction and PCA}

Traditional methods of spectral analysis use peak heights or peak areas, while PCA uses the whole spectrum. Because of the presence of spinning sidebands resulting from hardware restriction of the spinning rate to $2 \mathrm{kHz}$, and relatively few and signals in the aromatic region, only signals between $\delta 0.50$ and 4.50 were selected for PCA. Each sub-spectrum was divided into 4000 regions with a uniform width of $0.001 \mathrm{ppm}$. The signal intensities in each region were integrated, and each integrated region was normalized by the sum of all integrals over the entire sub-spectrum.

To determine if it was possible to distinguish between various grades of brain tumors using HRMAS ${ }^{1} \mathrm{H}$ NMR spectra data, PCA and partial least squares-discriminant analysis (PLS-DA) were carried out with mean-center scaling using SIMCA-P 10.0 software (Umetrics, Sweden). Processed data were visualized by score plots and regression coefficient (or loading) plots, where each point on the score plot represented the metabonome of an individual sample, while each bin on the regression coefficient (loading) plot represented a single spectral region (or chemical shift) contributing to the separation [27,30-32].

\subsection{Pattern recognition}

The classification and prediction of various grade tumors was maximized using a supervised pattern recognition method: Soft Independent Modeling of Class Analogy (SIMCA) was performed using various class information for tumors as defined " $Y$ ' variables. The data set was divided into "training" and "test" sets, and the principal components of each class in the training set were calculated. The class membership of set samples was assigned according to their position within the calculated boundaries of the training set group, specified in a Cooman's residual plot [27]. In addition, orthogonal signal correction (OSC) was used to optimize the separation [33]. An orthogonal partial least squares (OPLS) model was used to predict the grade classification of tumors. OPLS is an extension to the supervised PLS method featuring an integrated OSC filter to remove variability not relevant to class separation.

In the present study, all 30 tumor samples were initially assigned to two classes. All 14 samples from grade I and grade II astrocytomas constituted the low-grade class (class 1), whereas the remainder from grade III astrocytomas, glioblastomas and medulloblastomas constituted the highgrade class (class 2). Half of the samples from each class were selected as the 'training' set, while the remaining samples were used as the 'test' set. SIMCA models were 
established based on HRMAS ${ }^{1} \mathrm{H}$ NMR data (or filter data). When there were only a few samples in the dataset, the 'leave-one-out' method, in which each sample is excluded in turn from the 'training' data and used as the 'test' datum, was used to test the discriminant function.

\subsection{Statistical analysis}

The absolute concentrations of metabolites are difficult to quantify using HRMAS NMR spectra, and the relative concentrations were therefore defined as the ratio of the individual metabolite signal integrals over that of the total spectra. Every NMR peak was therefore normalized to the sum of the total spectrum, and the resulting data were used for statistical analysis.

The normalized integrals of some important metabolites for 30 human brain tumors and their ratios relative to Cre ( $\delta 3.93$ ) were subjected to two-tailed analysis of variance (ANOVA) using the statistical software SPSS 10.0 (Statistical Package for Social Science, SPSS Inc., USA). Changes in metabolite levels were considered to be statistically significant when $P<0.05$.

\section{Results}

\subsection{Pathological assessment and photomicrographs of tumor samples}

Figure 1 shows histological sections (H\&E stain) of normal tissue, grade I, II, and III astrocytomas, a glioblastoma (grade IV) and a medulloblastoma (grade IV). The number of tumor cells rapidly increased with increasing tumor grade. Compared with normal tissue (Figure 1A), the grade I-II astrocytomas (Figure 1B and C) showed clear increases in the number of tumor cells with no mitosis, while the grade III astrocytoma (Figure 1D) showed further increases in tumor cells accompanied by a visibly invasive schistosome ovum. The grade IV glioblastoma (Figure 1E), regarded as a grade IV astrocytoma, displayed marked endothelial proliferation and mitosis. The grade IV medulloblastoma (Figure
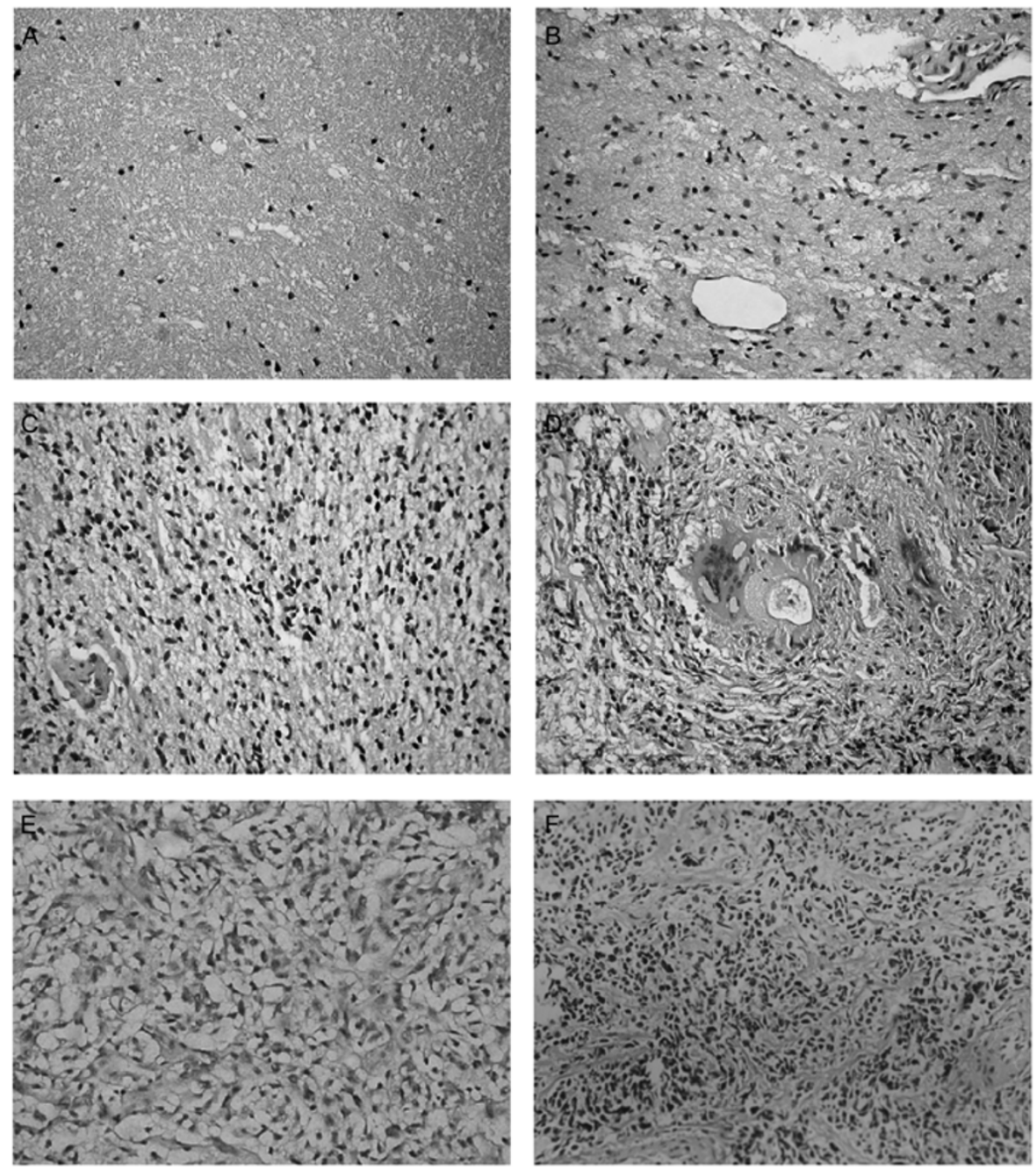

Figure 1 Photomicrographs (H\&E stain) of normal tissue and five brain tumor specimens. A, Normal tissue (200×). B, Astrocytoma (200×, grade I). C, Astrocytoma (200×, grade II). D, Astrocytoma (200×, grade III). E, Glioblastoma (200×, grade IV). F, Medulloblastoma (200×, grade IV). 
1F) was characterized by sheets of densely packed cells with more abundant cytoplasm and numerous mitoses.

\subsection{HRMAS ${ }^{1} \mathrm{H}$ NMR spectroscopy of brain tumors}

Figure 2 shows the HRMAS ${ }^{1} \mathrm{H}$ NMR spectra acquired using a CPMG pulse sequence at a spinning rate of $2 \mathrm{kHz}$, corresponding to the above H\&E samples. Several metabolite resonances were detected from the spectra. Spectral assignments were confirmed by ${ }^{1} \mathrm{H}-{ }^{1} \mathrm{H}$ total correlation spectroscopy HRMAS 2D NMR spectra (data not shown), together with published data [18,20,25,34-36]. For astrocytomas (Figure 2B-D), the levels of remainder Lip $(\delta 0.92)$, Lac $(\delta 1.33$ ), alanine (Ala, $\delta 1.48)$ and scyllo-inositol (sI, $\delta 3.35)$ increased with increasing tumor grade, while the levels of NAA ( $\delta 2.03)$, myo-inositol (mI, $\delta 3.62)$ and Cre $(\delta 3.04)$ decreased. Higher levels of glycine (Gly, $\delta 3.56$ ) and Lip $(\delta 0.92)$ together with lower levels of NAA, phosphocholine (PC, $\delta 3.22)$ and glycerophosphocholine (GPC, $\delta 3.23)$ were the principal characteristics of glioblastomas (Figure 2E). Higher levels of taurine (Tau, $\delta 3.43$ ), $\mathrm{PC}$ and GPC, together with lower levels of Lac, were the chief characteristics of medulloblastomas (Figure 2F).

Figure 3 shows the average HRMAS ${ }^{1} \mathrm{H}$ NMR spectra $($ mean \pm SD) for the five groups of tumor tissues with various grades recorded by CPMG pulse sequences. Apart from the intense fatty acid signals, which were not completely suppressed by the CPMG pulse sequence because of the short relaxation time $(32 \mathrm{~ms})$, a number of metabolite differences
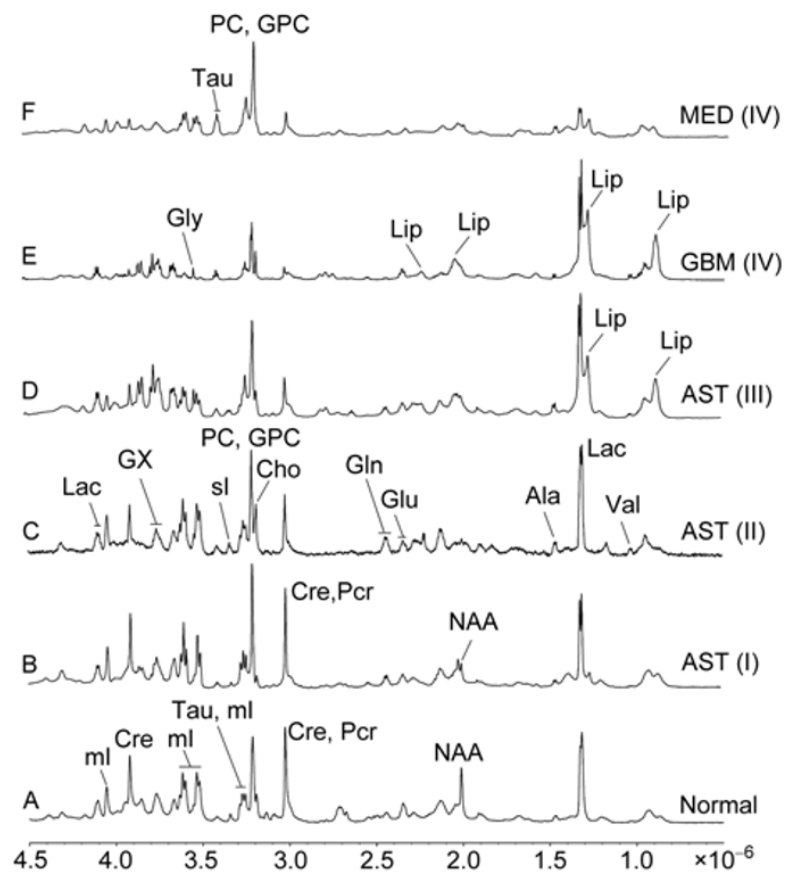

Figure 2 HR-MAS ${ }^{1} \mathrm{H}$ NMR spectra of six brain specimens acquired from CPMG pulse sequence at a spin rate of $2 \mathrm{kHz}$. A, Normal tissue. B, Astrocytoma (grade I). C, Astrocytoma (grade II). D, Astrocytoma. E, Glioblastoma (grade IV). F, Medulloblastoma (grade IV).
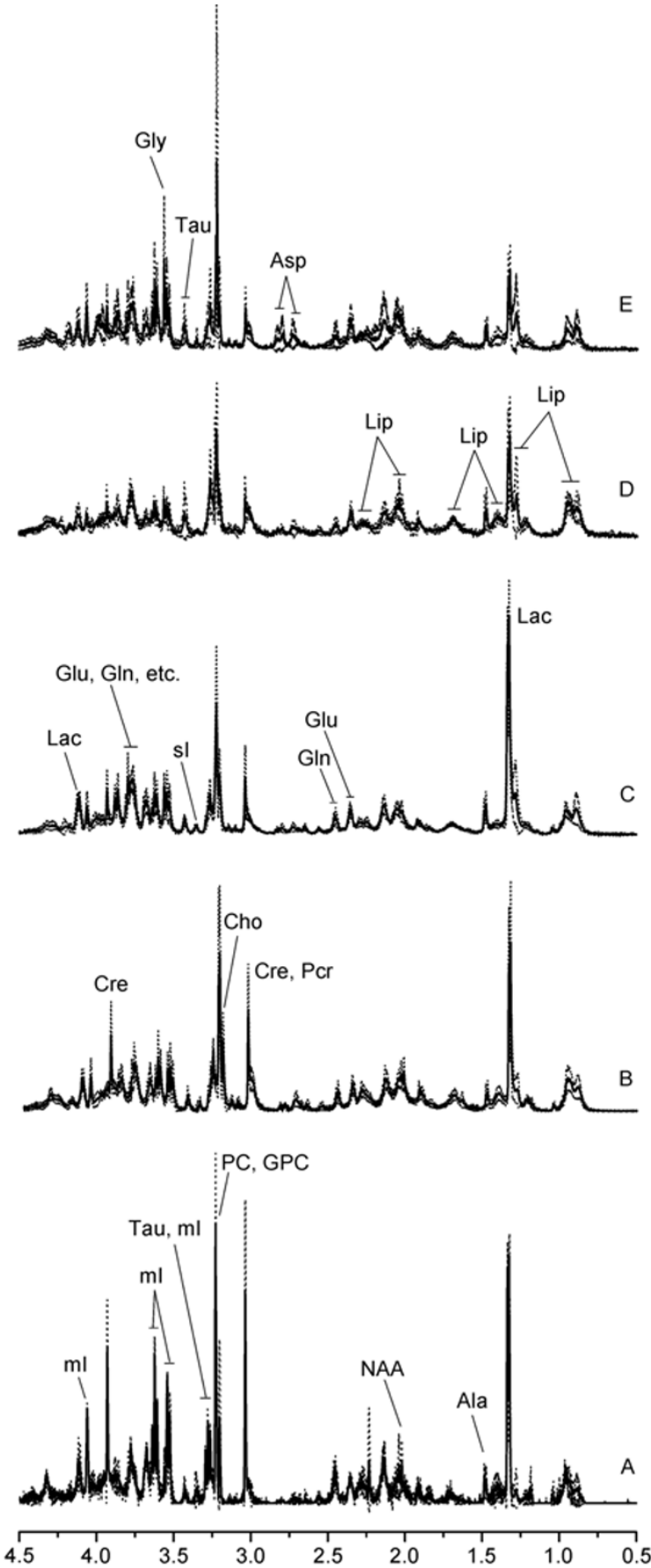

Figure 3 Average HRMAS ${ }^{1} \mathrm{H}$ NMR spectra for 30 tumor samples obtained by CPMG pulse sequence at a spinning rate of $2 \mathrm{kHz}$. A, Astrocytomas (grade I). B, Astrocytomas (grade II). C, Astrocytomas (grade III). D, Glioblastomas (grade IV). E, Medulloblastomas (grade IV). *, The real line and dashed line represent mean and standard deviation (SD) of the spectra for each grade group, respectively.

could be detected between the spectra of the various grades of tumors. The levels of Lip and Ala in grade II and III astrocytomas were higher than in grade I astrocytomas (Figure 3A), while the levels of NAA, mI, Cre, PC and GPC were lower (Figure 3B and C). The levels of Lip and Tau in grade IV glioblastomas were higher than in grade I tumors, but the levels of Lac and Cre were much lower (Figure 3D). How- 
ever, compared with grade II-III astrocytomas and grade IV glioblastomas, the levels of $\mathrm{mI}$, Tau, Gly, PC, GPC and aspartate (Asp) in grade IV medulloblastomas were relatively high, whereas the levels of Lac and Cre were low (Figure 3E).

\subsection{PCA of HRMAS ${ }^{1}$ H NMR data}

Figure 4 shows PLS-DA score plots and regression coefficient plots of HRMAS ${ }^{1} \mathrm{H}$ NMR spectra for various groups of tumors. The first principal component (PC1) in Figure $4 \mathrm{~A}$ accounted for $24.4 \%$ of all the variations in the MAS spectra, and showed good separation between grade I-II (group 1-2) and grade III (group 3) astrocytomas. Correspondingly, the regression coefficients in Figure 4B indicate that the levels of NAA, Cre, GPC and $\mathrm{mI}$ in grade I-II astrocytomas (group 1-2) were relatively high but the levels of Lac and PC were lower, compared with grade III astrocytomas (group 3). The PC1 in Figure 4C explained 27.8\% of all the variations in the MAS spectra and also showed good separation between grade I-II (group 1-2) and grade IV (group 4-5) tumors. Compared with grade IV tumors, the positive values of the regression coefficients in Figure 4D indicate that the levels of Lac, Cre, Cho and GPC in grade I-II tumors were higher, whilst the negative values indicated that the levels of Gly and PC were lower. Similarly, the PC1 in Figure 4E (explaining 29.8\% variations) showed a good separation between grade III (group 3) and grade IV (group 4-5) tumors. The regression coefficients in Figure 4F show that the levels of Lac, Cre, Cho and GPC in grade III astrocytomas (group 3) were higher, but the levels of $\mathrm{mI}$ and PC were lower, compared with grade IV tumors (group 4-5). Finally, the PC1 in Figure 4G (explaining $23.2 \%$ variation) also showed good separation between glioblastomas (group 4) and medulloblastomas (group 5), whereas the PC1 in Figure 4I (explaining 33.3\% variation) showed good separation between grade I (group 1) and grade II astrocytomas (group 2). The regression coefficients of $\mathrm{PC} 1$ (Figure $4 \mathrm{H}$ and $\mathrm{J}$ ) showed the important contributions of some metabolites to the differentiation between groups.

\subsection{SIMCA analysis of HRMAS ${ }^{1} \mathrm{H}$ NMR data}

Figure 5 shows an OPLS model that can be used to predict the class membership of the tumor samples. The PC1 accounted for $66 \%$ of all the variations in the spectra, and showed a clear separation between class 1 and class 2 . The OPLS score plot was characterized by the following parameters: $R^{2} Y=0.96$ and $Q^{2}=0.95$. The high $Q^{2}$ value indicated the good predictive capabilities of the model.

Figure 6 shows the Cooman residual plot for membership of class 1 and class 2 . This plot is divided into four regions based on the $95 \%$ confidence limits for both classes. Six of seven tested tumor samples in class 1 (upper left region) were correctly classified as low-grade, while seven of eight tested tumor samples in class 2 (lower right region) were classified as high-grade. The accuracy of each class prediction using the SIMCA model was almost $87 \%$. Furthermore, the present SIMCA model predicted the presence of grade III-IV brain tumors (class 2) with a sensitivity of $87 \%$ and a specificity of $93 \%$. The classification results using the "leave-one-out" method are summarized in Table 2. For instance, the percentage of each class correctly classified for filter data was $100 \%$.

\subsection{Statistical analysis}

Changes in metabolite levels and metabolite/Cre ratios (Figures 3 and 4) were analyzed by two-tailed ANOVA, and the results are summarized in Tables 3 and 4. As shown in Table 3, the levels of some metabolites gradually decreased with increasing tumor grade (i.e., from grade I to grade IV), whereas the levels of other metabolites gradually increased with increasing tumor grade. These metabolites mainly included mI, Cre, PC, GPC, Gly, Tau and Asp. Furthermore, significant differences $(P<0.05)$ in levels of NAA, Cre, mI, Gly and Lac were observed between groups (Table 3). No significant differences in levels of Tau, sI, Asp, Glu, Ala, valine, Lip or tCho (Cho, PC and GPC) were found. There were significant differences in NAA/Cre, Lac/Cre, mI/Cre, Gly/Cre, sI/Cre and Ala/Cre ratios between groups $(P<0.05)$ (Table 4).

\section{Discussion}

The poor survival rate of patients with malignant brain tumors means that improved understanding leading to their early detection and accurate grade classification is vitally important in terms of determining their prognosis and appropriate treatment. In the present study, we observed clear differences between HRMAS ${ }^{1} \mathrm{H}$ NMR spectra correlated with the histopathological specimens. However, the complexity of the spectra and the large number of samples make simple visual inspection difficult, and small differences in spectra between different samples in the same group may be especially difficult because of microheterogeneity among individuals [37] and/or inconsistence of sampling sites. Effective methods for rapidly observing differences between spectra in large numbers of tumor tissue samples are therefore needed.

This study therefore used two methods, average spectra and PCA of various group samples, to maximize the scope for differentiating among tumors and classifying tumors. Changes in levels of some metabolites, including Lac, mI, Cre, Gly and tCho (Cho, PC and GPC), were clearly demonstrated between different groups by examining average spectra. Regression coefficient plots of PLS-DA showed the important contributions of some metabolites to the separa- 

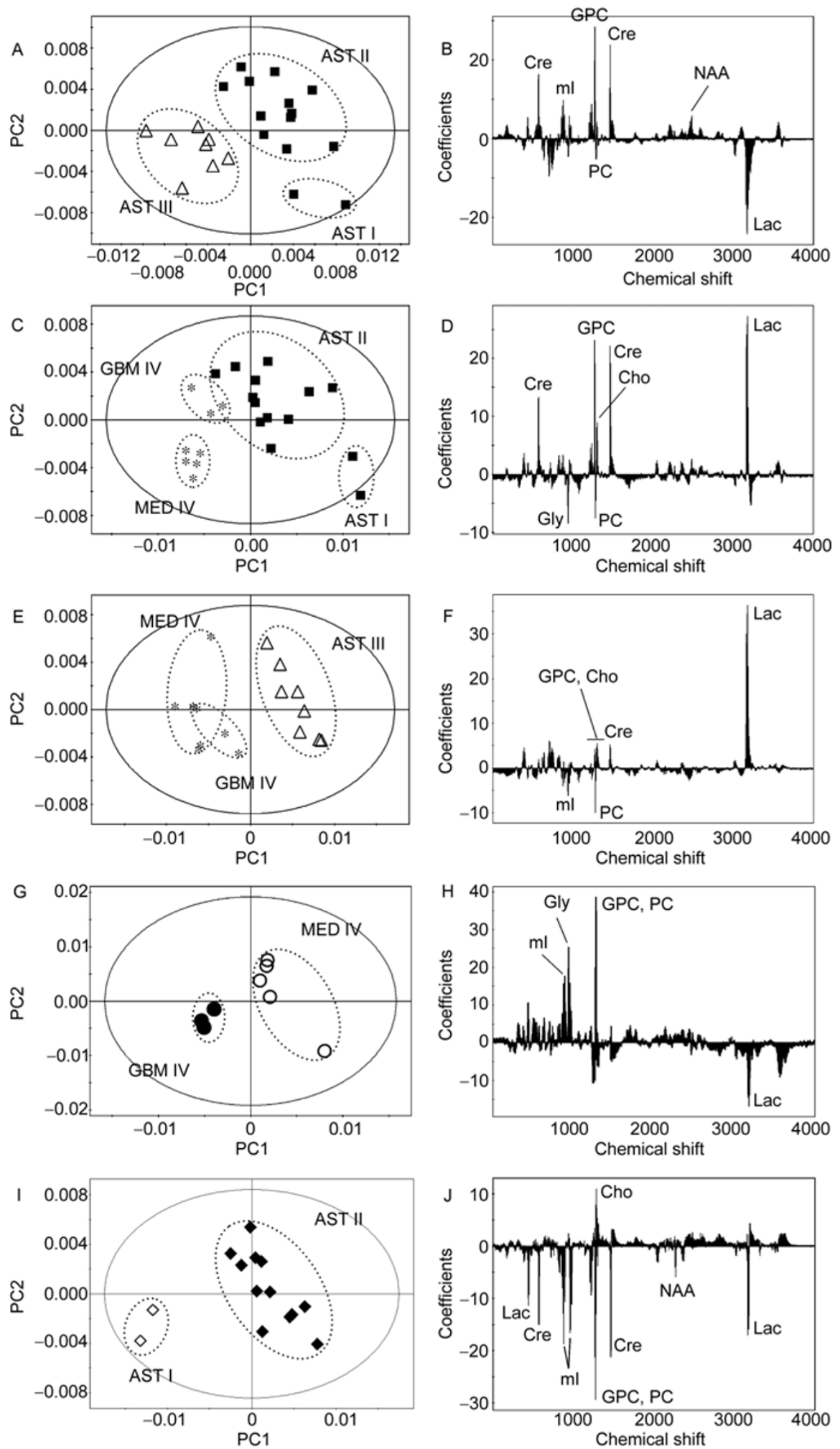

Figure 4 Comparison of PLS-DA score plots and regression coefficient plots of HRMAS ${ }^{1} \mathrm{H}$ NMR spectra from 30 tumor samples between different grade tumors. A and B, PLS-DA score plot (A) and regression coefficient plot (B) of PC1, respectively, produced using only groups 1-2 and 3. C and D, PLS-DA score plot (C) and regression coefficient plot (D) of PC1, respectively, produced using only groups 1-2 and 4-5. E and F, PLS-DA score plot (E) and regression coefficient plot (F) of PC1, respectively, produced using only groups 3 and 4-5. G and H, PLS-DA score plot (G) and regression coefficient plot (H) of PC1, respectively, produced using only GBM and MED. I and J, PLS-DA score plot (I) and regression coefficient plot (J) of PC1, respectively, produced using only AST I and AST II. $\mathbf{\square}$, Group 1-2; $\triangle$, group 3; *, group 4-5;, GBM (glioblastoma); $\bigcirc$, MED (medulloblastoma); $\diamond$, AST I (astrocytoma I); 


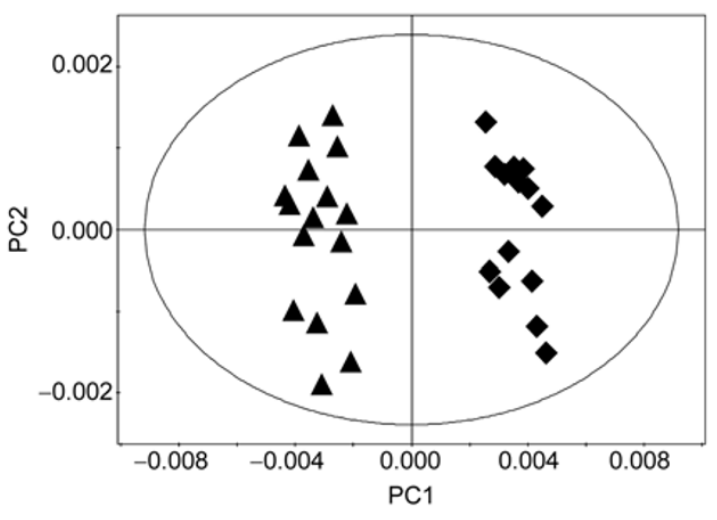

Figure 5 PC1 vs. PC2 score plots of filtered-PLS model obtained from HR MAS ${ }^{1} \mathrm{H}$ NMR spectra of 30 tumors samples. $\bullet$, Low-grade (I-II) brain tumors; $\mathbf{\Lambda}$, high-grade (III-IV) brain tumors.

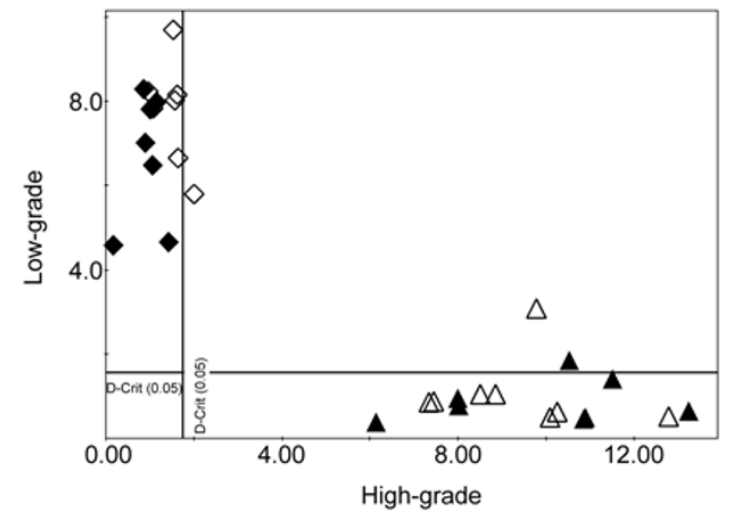

Figure 6 Cooman plot of HR MAS ${ }^{1} \mathrm{H}$ NMR spectra for 30 tumor samples from low-grade brain tumors from the "training" set $(\bullet)$ and the "test" set $(\diamond)$, and high-grade brain tumors from the "training" set $(\boldsymbol{\Delta})$ and the "test" set $(\triangle)$.

Table 2 Classification results for the various models before and after OSC filter data ${ }^{\text {a) }}$

\begin{tabular}{ccccccc}
\hline \multirow{2}{*}{ Grade class } & \multirow{2}{*}{ Total cases } & \multicolumn{2}{c}{ Number of cases assigned to each class (PLS model) $^{*}$} & & Number of cases assigned to each class (OPLS model) $^{\#}$ \\
\cline { 3 - 5 } & & Class 1 & 12 & 2 & Class 1 & Class 2 \\
\hline Class 1 & 14 & 0 & 16 & 14 & 0 \\
Class 2 & 16 & 0 & 0 & 16 \\
\hline
\end{tabular}

a) *, Percentage of classes correctly classified: $93 \%$ (28/30); \#, percentage of classes correctly classified: 100\% (30/30).

Table 3 Selected metabolite concentrations (percentages of normalized integrals) calculated from HRMAS ${ }^{1} \mathrm{H}$ NMR spectra for differentiating between different grade tumors ${ }^{\text {a }}$

\begin{tabular}{|c|c|c|c|c|c|c|}
\hline Metabolite (chemical shift) & $\begin{array}{c}\text { AST I }(n=2) \\
\text { Mean } \pm \text { SD }\end{array}$ & $\begin{array}{c}\text { AST II }(n=12) \\
\text { Mean } \pm \text { SD }\end{array}$ & $\begin{array}{c}\text { AST III }(n=8) \\
\text { Mean } \pm \text { SD }\end{array}$ & $\begin{array}{c}\text { GBM }(n=3) \\
\text { Mean } \pm \text { SD }\end{array}$ & $\begin{array}{c}\text { MED }(n=5) \\
\text { Mean } \pm \text { SD }\end{array}$ & $P$-value* \\
\hline NAA $(\delta 4.38)$ & $1.26 \pm 0.25$ & $0.90 \pm 0.24$ & $0.56 \pm 0.21$ & $0.88 \pm 0.31$ & $0.91 \pm 0.22$ & $<0.01$ \\
\hline lactate $(\delta 4.12)$ & $1.90 \pm 0.22$ & $1.46 \pm 0.26$ & $1.74 \pm 0.28$ & $1.16 \pm 0.44$ & $1.26 \pm 0.40$ & $<0.05$ \\
\hline creatine $(\delta 3.93)$ & $2.76 \pm 0.59$ & $1.64 \pm 0.55$ & $1.10 \pm 0.44$ & $0.90 \pm 0.38$ & $1.04 \pm 0.38$ & $<0.001$ \\
\hline myo-inositol $(\delta 3.62)$ & $5.75 \pm 0.50$ & $2.94 \pm 0.86$ & $2.64 \pm 0.80$ & $2.17 \pm 0.45$ & $3.62 \pm 1.20$ & $<0.001$ \\
\hline glycine $(\delta 3.56)$ & $0.67 \pm 0.32$ & $0.77 \pm 0.18$ & $0.93 \pm 0.23$ & $0.69 \pm 0.06$ & $1.37 \pm 0.74$ & $<0.05$ \\
\hline taurine $(\delta 3.43)$ & $0.41 \pm 0.18$ & $0.47 \pm 0.19$ & $0.51 \pm 0.15$ & $0.76 \pm 0.82$ & $0.81 \pm 0.47$ & $0.20-0.30$ \\
\hline scyllo-inositol $(\delta 3.35)$ & $0.52 \pm 0.47$ & $0.33 \pm 0.15$ & $0.44 \pm 0.11$ & $0.31 \pm 0.08$ & $0.43 \pm 0.21$ & $0.40-0.50$ \\
\hline $\mathrm{PC}+\mathrm{GPC}(\delta 3.22)$ & $5.61 \pm 0.12$ & $4.88 \pm 1.64$ & $4.18 \pm 1.13$ & $3.59 \pm 0.37$ & $4.63 \pm 2.82$ & $0.60-0.70$ \\
\hline choline $(\delta 3.20)$ & $1.53 \pm 1.36$ & $1.82 \pm 0.71$ & $1.71 \pm 0.63$ & $1.33 \pm 0.26$ & $1.07 \pm 0.68$ & $0.30-0.40$ \\
\hline tCho $(\delta 3.21)$ & $7.14 \pm 1.25$ & $6.69 \pm 2.06$ & $5.89 \pm 1.45$ & $4.92 \pm 0.50$ & $5.70 \pm 3.39$ & $0.60-0.70$ \\
\hline aspartate $(\delta 2.82)$ & $0.06 \pm 0.08$ & $0.18 \pm 0.07$ & $0.24 \pm 0.11$ & $0.26 \pm 0.12$ & $0.48 \pm 0.47$ & $<0.08$ \\
\hline glutamine $(\delta 2.46)$ & $1.34 \pm 0.49$ & $0.87 \pm 0.37$ & $0.91 \pm 0.35$ & $0.66 \pm 0.37$ & $0.75 \pm 0.54$ & $0.40-0.50$ \\
\hline glutamate ( $\delta 2.35)$ & $1.09 \pm 0.08$ & $1.24 \pm 0.34$ & $1.21 \pm 0.25$ & $1.19 \pm 0.30$ & $1.40 \pm 0.57$ & $0.80-0.90$ \\
\hline alanine $(\delta 1.48)$ & $0.69 \pm 0.33$ & $0.55 \pm 0.29$ & $0.89 \pm 0.33$ & $0.85 \pm 0.46$ & $0.70 \pm 0.16$ & $0.10-0.20$ \\
\hline valine $(\delta 1.05)$ & $0.19 \pm 0.21$ & $0.21 \pm 0.07$ & $0.26 \pm 0.10$ & $0.19 \pm 0.05$ & $0.19 \pm 0.11$ & $0.60-0.70$ \\
\hline lipid $(\delta 0.92)$ & $3.18 \pm 1.32$ & $4.52 \pm 1.48$ & $3.90 \pm 1.02$ & $5.28 \pm 1.52$ & $3.29 \pm 1.44$ & $0.20-0.30$ \\
\hline
\end{tabular}

a) Abbreviations: NAA, N-acetyl aspartate; PC, phosphocholine; GPC, glycerophosphocholine; tCho, includes choline, PC and GPC; AST, astrocytoma; GBM, glioblastoma; MED, medulloblastoma. *, $P<0.05$, two-tailed ANOVA method.

tion of groups. The present HRMAS study thus provides more information on metabolites correlated with the histopathology of tissue specimens, increasing the chances of identifying key molecular pathways and so improving our understandings of tumor biology. This in turn will aid the accurate classification and grading of human brain tumors. The relationships between changes in metabolites and grade classification of brain tumors are elucidated below.

Some studies have reported the use of in vivo MRS, and in vitro NMRS and MAS NMRS to distinguish between various grades of brain tumors, and the results are summarized in Table 5. The limited spectral resolution and detection sensitivity of in vivo ${ }^{1} \mathrm{H}$ MRS studies [14-17,34,38], mean that signals for Cho, PC and GPC were often collec- 
tively reported as "tCho", while the signals for Glu and Gln were often reported together as $\operatorname{GLX}(\delta 2.4,3.7)$. Signals for Gly ( $\delta 3.56)$ might be mistakenly assigned as mI. However, the present MAS study resolved the signals for Glu ( $\delta 2.35)$ and Gln $(\delta 2.46)$, allowing them to be considered separately, while PC $(\delta 3.22)$ and GPC $(\delta 3.23)$ could be resolved using curve-fitting methods. The significant changes in NAA, Cre, mI, Gly and Lac were consistent with the above in vivo studies, and the significant differences in metabolite/creatine ratios between groups were also consistent with these studies, except in the case of sI/Cre, which was observed for the first time (Tables 4 and 5). No significant differences in tCho, Ala and Lip levels were found, possibly because the levels of PC, GPC and Lip decreased (and/or increased) as a result of necrosis [14,39,40] in high-grade tumors, such as glioblastomas.

Previous in vitro ${ }^{1} \mathrm{H}$ NMRS studies [36,41,42] found no significant differences in NAA and Cre levels [36,41] between lower- and higher-grade brain tumors. No information on lipid levels was reported, because traditional tissue extraction methods usually remove lipophilic lipids. The current study thus detected significant differences in more metabolite concentrations and ratios than previous in vitro studies [36,42] (Table 5).

Finally, in ex vivo MAS studies, Cheng et al. [43] only observed significant differences in Cho $(\delta 3.20)$ levels and the $\mathrm{mI} / \mathrm{Cre}$ ratios $(\delta 4.05)$ between three astrocytomas and eight glioblastomas. Furthermore, the signal peak ( $\delta$ 4.05) was simply assigned to inositol, though the signals were mainly generated by Cho and mI. Wilson et al. [44] recently found significant differences in levels of Cre, Gln, Tau, phosphoethanolamine $(\delta 3.98)$ and $\mathrm{PC}$ between 12 glial tumors (10 pilocytic astrocytomas and two ependymomas) and 17 primitive neuroectodermal tumors (nine medulloblastomas, seven neuroblastomas, and one supratentorial primitive neuroectodermal tumor); however, all the tissue samples in their study were resected from 29 pediatric patients, while most of the samples in the current study were from adults. The present study thus detected significant differences in levels and ratios of more metabolites between groups, compared with previous MAS studies [43,44] (Table 5). This suggests that HRMAS ${ }^{1} \mathrm{H}$ NMRS can act as a valuable adjunct to current neuropathological techniques and may increase the diagnostic accuracy of human brain tumors.

Regarding the grade classification of brain tumors, some studies have reported relatively high predictive abilities of NMRS combined with PR methods such as linear discriminant analysis [16,17,41, 44,45] and neural networks [18] for differentiating between various tumors. Compared with

Table 4 Selected metabolite/creatine ratios $(\delta 3.93)$ calculated from HRMAS ${ }^{1} \mathrm{H}$ NMR spectra for differentiating between different grade tumors ${ }^{\text {a) }}$

\begin{tabular}{|c|c|c|c|c|c|c|}
\hline Metabolite ratio (chemical shift) & $\begin{array}{c}\text { AST I }(n=2) \\
\text { Mean } \pm \text { SD }\end{array}$ & $\begin{array}{c}\text { AST II }(n=12) \\
\text { Mean } \pm \text { SD }\end{array}$ & $\begin{array}{c}\text { AST III }(n=8) \\
\text { Mean } \pm \text { SD }\end{array}$ & $\begin{array}{c}\text { GBM }(n=3) \\
\text { Mean } \pm \text { SD }\end{array}$ & $\begin{array}{c}\operatorname{MED}(n=5) \\
\text { Mean } \pm \text { SD }\end{array}$ & $P$-value ${ }^{*}$ \\
\hline NAA/Cre $(\delta 4.38)$ & $0.46 \pm 0.01$ & $0.61 \pm 0.28$ & $0.56 \pm 0.19$ & $1.11 \pm 0.54$ & $0.94 \pm 0.29$ & $<0.05$ \\
\hline lactate/Cre $(\delta 4.12)$ & $0.71 \pm 0.23$ & $0.97 \pm 0.34$ & $1.76 \pm 0.59$ & $1.55 \pm 1.20$ & $1.33 \pm 0.58$ & $<0.05$ \\
\hline myo-inositol/Cre $(\delta 3.62)$ & $2.15 \pm 0.64$ & $1.88 \pm 0.51$ & $2.61 \pm 0.90$ & $2.63 \pm 1.04$ & $3.85 \pm 1.95$ & $<0.05$ \\
\hline glycine/Cre $(\delta 3.56)$ & $0.26 \pm 0.17$ & $0.53 \pm 0.26$ & $0.96 \pm 0.41$ & $0.88 \pm 0.41$ & $1.65 \pm 1.54$ & $<0.05$ \\
\hline taurine/Cre $(\delta 3.43)$ & $0.16 \pm 0.10$ & $0.35 \pm 0.33$ & $0.50 \pm 0.18$ & $1.22 \pm 1.65$ & $0.82 \pm 0.41$ & $0.10-0.20$ \\
\hline$(\mathrm{PC}+\mathrm{GPC}) / \mathrm{Cre}(\delta 3.22)$ & $2.08 \pm 0.40$ & $3.17 \pm 1.19$ & $4.07 \pm 1.13$ & $4.62 \pm 2.47$ & $5.49 \pm 5.02$ & $0.30-0.40$ \\
\hline choline/Cre $(\delta 3.20)$ & $0.62 \pm 0.63$ & $1.20 \pm 0.53$ & $1.79 \pm 1.00$ & $1.65 \pm 0.81$ & $1.35 \pm 1.40$ & $0.40-0.50$ \\
\hline $\mathrm{tCho} / \mathrm{Cre}(\delta 3.21)$ & $2.70 \pm 1.03$ & $4.38 \pm 1.53$ & $5.85 \pm 2.01$ & $6.27 \pm 3.26$ & $6.83 \pm 6.37$ & $0.30-0.40$ \\
\hline aspartate/Cre $(\delta 2.82)$ & $0.02 \pm 0.02$ & $0.14 \pm 0.11$ & $0.25 \pm 0.16$ & $0.34 \pm 0.21$ & $0.66 \pm 0.87$ & $0.10-0.20$ \\
\hline glutamine/Cre $(\delta 2.46)$ & $0.52 \pm 0.29$ & $0.59 \pm 0.39$ & $0.87 \pm 0.26$ & $0.79 \pm 0.44$ & $0.69 \pm 0.26$ & $0.40-0.50$ \\
\hline glutamate/Cre $(\delta 2.35)$ & $0.41 \pm 0.12$ & $0.88 \pm 0.62$ & $1.25 \pm 0.52$ & $1.47 \pm 0.60$ & $1.53 \pm 0.88$ & $0.10-0.20$ \\
\hline alanine/Cre $(\delta 1.48)$ & $0.27 \pm 0.18$ & $0.42 \pm 0.39$ & $0.96 \pm 0.55$ & $1.15 \pm 0.77$ & $0.75 \pm 0.32$ & $<0.05$ \\
\hline valine/Cre $(\delta 1.05)$ & $0.08 \pm 0.09$ & $0.15 \pm 0.10$ & $0.28 \pm 0.19$ & $0.24 \pm 0.11$ & $0.19 \pm 0.15$ & $0.20-0.30$ \\
\hline
\end{tabular}

a) Abbreviations: Cre, creatine; NAA, N-acetyl aspartate; PC, phosphocholine; GPC, glycerophosphocholine; tCho, includes choline, PC and GPC; AST, astrocytoma; GBM, glioblastoma; MED, medulloblastoma. *, $P<0.05$, two-tailed ANOVA method.

Table 5 Concentrations and ratios of metabolites showing significant differences between different grade tumors

\begin{tabular}{|c|c|c|}
\hline NMR method & Significantly different concentrations and ratios of metabolite $(P<0.05)$ & Literature \\
\hline In vivo $\mathrm{MRS}$ & $\begin{array}{c}\text { NAA, Ala, Cre, tCho, mI ( } \delta 3.56) \text {, Lac, Glu (or Gln, } \delta 3.7) \text {, Lip ( } \delta 1.3), \text { NAA/Cre, } \\
\text { tCho/Cre, Lac/Cre, Gly/Cre, mI/tCho, mI/Cre, Ala/Cre }\end{array}$ & $\begin{array}{l}\text { Howe et al. [34], Tong et al. [14], Fayed et al. } \\
\text { [15], Majos et al. [16,17], Meyerand et al. [38] }\end{array}$ \\
\hline In vitro $\mathrm{NMR}$ & Ala, tCho, Tau, mI, Gly, Glu, Gly/Cre, Ala/Cre, tCho/Cre, Glu/Gln & $\begin{array}{c}\text { Kinoshita \& Yokota [36], Roda et al. }[41] \\
\text { Lehnhardt } \text { et al. } \text { [42] }\end{array}$ \\
\hline Ex vivo MAS & Cre, Gln, Tau, PC, PE, Cho, mI ( $\delta 4.06) /$ Cre & Cheng et al. [43], Wilson et al. [44] \\
\hline Ex vivo MAS & NAA, Lac, Cre, mI, Gly, NAA/Cre, Lac/Cre, mI/Cre, Gly/Cre, sI/Cre, Ala/Cre & Our study \\
\hline
\end{tabular}


previous published studies on in vivo MRS [16,17,45], in vitro NMRS [18,41] and ex vivo MAS NMRS [44], the present study demonstrated that HRMAS ${ }^{1} \mathrm{H}$ NMR spectroscopy combined with pattern recognition can increase the accuracy of grade classification and diagnosis of neuroepithelial tumors, especially using the "leave-one-out" method. Further studies with larger sample sizes are needed to verify these findings.

\section{Conclusion}

HRMAS ${ }^{1} \mathrm{H}$ NMR spectroscopy combined with pattern recognition can provide valuable metabonomic information on metabolites relevant to understanding the biology and pathology of brain tumors. It can also provide a potentially useful tool for the rapid and accurate grade classification of human brain tumors, which will help histopathologists to classify brain tumors more accurately, ultimately leading to the development of automatic diagnostic methods.

This work was supported by the National Natural Science Foundation of China (Grant Nos. 20573132 and 20575074), China Postdoctoral Science Foundation (Grant No. 20090450065) and State Key Laboratory of Magnetic Resonance and Atomic and Molecular Physics (Grant No. T152805).

1 Mertens A C, Yasui Y, Neglia J P, et al. Late mortality experience in five-year survivors of childhood and adolescent cancer: the childhood cancer survivor study. J Clin Oncol, 2001, 19: 3163-3172

2 Mainio A, Hakko H, Timonen M, et al. Depression in relation to survival among neurosurgical patients with a primary brain tumor: a 5-year follow-up study. Neurosurgery, 2005, 56: 1234-1241

3 Sant M, Van der Sanden G, Capocaccia R. Survival rates for primary malignant brain tumours in Europe. EUROCARE Working Group. Eur J Cancer, 1998, 34: 2241-224

4 Barnholtz-Sloan J S, Sloan A E, Schwartz A G. Relative survival rates and patterns of diagnosis analyzed by time period for individuals with primary malignant brain tumor, 1973-1997. J Neurosurgery, 2003, 99: 458-466

5 Iwadate Y, Sakaida T, Hiwasa T, et al. Molecular classification and survival prediction in human gliomas based on proteome analysis. Cancer Res, 2004, 64: 2496-250

6 Engelhard H H, Stelea A, Cochran E J. Oligodendroglioma: pathology and molecular biology. Surg Neurol, 2002, 58: 111-117

7 Rickert C H, Paulus W. Prognosis-related histomorphological and immunohistochemical markers in central nervous system tumors of childhood and adolescence. Acta Neuropathol, 2005, 109: 69-92

8 Zarbo R J, Meier F A, Raab S S. Error detection in anatomic pathology. Arch Pathol Lab Med, 2005, 129: 1237-1245

9 Salpietro F M, Alafaci C, Lucerna S, et al. Peritumoral edema in meningiomas microsurgical observations of different brain tumor interfaces related to computed tomography. Neurosurgery, 1994, 35: 638-641

10 Simonetti A W, Melssen W J, Szabo de Edelenyi F, et al. Combination of feature-reduced MR spectroscopic and MR imaging data for improved brain tumor classification. NMR Biomed, 2005, 18: 34-43

11 Devos A, Simonetti A W, Van der Graaf M, et al. The use of multivariate MR imaging intensities versus metabolic data from MR spectroscopic imaging for brain tumour classification. J Magn Reson, 2005, 173: 218-228

12 Henze M, Mohammed A, Schlemmer H P, et al. PET and SPECT for detection of tumor progression in irradiated low-grade astrocytoma: a receiver-operating-characteristic analysis. J Nucl Med, 2004, 45: 579-586

13 Roelcke U, Leenders K L. PET in neuro-oncology. J Cancer Res Clin Oncol, 2001, 127: 2-8

14 Tong Z, Yamaki T, Harada K, et al. In vivo quantification of the metabolites in normal brain and brain tumors by proton MR spectroscopy using water as an internal standard. Magn Reson Imaging, 2004, 22: 1017-1024

15 Fayed N, Morales H, Modrego P J, et al. Contrast/noise ratio on conventional MRI and choline/creatine ratio on proton MRI spectroscopy accurately discriminate low-grade from high-grade cerebral gliomas. Acad Radiol, 2006, 13: 728-737

16 Majos C, Alonso J, Aguilera C, et al. Proton magnetic resonance spectroscopy $\left({ }^{1} \mathrm{H}\right.$ MRS $)$ of human brain tumours: assessment of differences between tumour types and its applicability in brain tumour categorization. Eur Radiol, 2003, 13: 582-591

17 Majos C, Julia-Sape M, Alonso J, et al. Brain tumor classification by proton MR spectroscopy: comparison of diagnostic accuracy at short and long TE. AJNR Am J Neuroradiol, 2004, 25: 1696-1704

18 Maxwell R J, Martinez-Perez I, Cerdan S, et al. Pattern recognition analysis of ${ }^{1} \mathrm{H}$ NMR spectra from perchloric acid extracts of human brain tumor biopsies. Magn Reson Med, 1998, 39: 869-877

19 Lehnhardt F G, Bock C, Rohn G, et al. Metabolic differences between primary and recurrent human brain tumors: a ${ }^{1} \mathrm{H}$ NMR spectroscopic investigation. NMR Biomed, 2005, 18: 371-382

20 Tugnoli V T M, Bertoluzza A, Barbarella G, et al. In vitro magnetic resonance spectroscopy of health and neoplastic brain tissues. J Mol Struct, 1999, 482: 365-369

21 Peeling J, Sutherland G. High-resolution ${ }^{1} \mathrm{H}$ NMR spectroscopy studies of extracts of human cerebral neoplasms. Magn Reson Med, 1992, 24: 123-136

22 Wilson M, Davies N P, Grundy R G, et al. A quantitative comparison of metabolite signals as detected by in vivo MRS with ex vivo ${ }^{1} \mathrm{H}$ HR-MAS for childhood brain tumours. NMR Biomed, 2009, 22: 213-219

23 Yang Y, Li C, Nie X, et al. Metabonomic studies of human hepatocellular carcinoma using high-resolution magic-angle spinning ${ }^{1} \mathrm{H}$ NMR spectroscopy in conjunction with multivariate data analysis. J Proteome Res, 2007, 6: 2605-2614

24 Righi V, Roda J M, Paz J, et al. ${ }^{1} \mathrm{H}$ HR-MAS and genomic analysis of human tumor biopsies discriminate between high and low grade astrocytomas. NMR Biomed, 2009, 22: 629-637

25 Chen W X, Lou H Y, Zhang H P, et al. Metabonomic characterization of the low-grade human astrocytomas and meningiomas using magic-angle spinning ${ }^{1} \mathrm{H}$ Nuclear magnetic resonance spectroscopy and principal component analysis. Prog Biochem Biophys, 2008, 35: 1142-1153

26 Waters N J, Garrod S, Farrant R D, et al. High-resolution magic angle spinning ${ }^{1} \mathrm{H}$ NMR spectroscopy of intact liver and kidney: optimization of sample preparation procedures and biochemical stability of tissue during spectral acquisition. Anal Biochem, 2000, 282: 16-23

27 Wang Y, Holmes E, Nicholson J K, et al. Metabonomic investigations in mice infected with Schistosoma mansoni: an approach for biomarker identification. Proc Natl Acad Sci USA, 2004, 101: 12676-12681

28 Cheng L L, Burns M A, Taylor J L, et al. Metabolic characterization of human prostate cancer with tissue magnetic resonance spectroscopy. Cancer Res, 2005, 65: 3030-3034

29 Nicholls A W, Mortishire-Smith R J. Temperature calibration of a high-resolution magic-angle spinning NMR probe for analysis of tissue samples. Magn Reson Chem, 2001, 39: 773-776

30 Wang Y, Bollard M E, Keun H, et al. Spectral editing and pattern recognition methods applied to high-resolution magic-angle spinning ${ }^{1} \mathrm{H}$ nuclear magnetic resonance spectroscopy of liver tissues. Anal Biochem, 2003, 323: 26-32

31 Eriksson L J E, Kettanah-Wold N. Introduction to Multi and Megavariate Data Analysis Using Projection Methods (PCA and PLS-DA) 1992-2002. Sweden: Umetrics AB, 2002

32 Brindle J T, Antti H, Holmes E, et al. Rapid and noninvasive diagnosis of the presence and severity of coronary heart disease using 
${ }^{1}$ H-NMR-based metabonomics. Nat Med, 2002, 8: 1439-1444

33 Wold S, Antti H, Lindgren F, et al. Orthogonal signal correction of near-infrared spectra. Chemometr Intell Lab, 1998, 44: 175-185

34 Howe F A, Barton S J, Cudlip S A, et al. Metabolic profiles of human brain tumors using quantitative in vivo ${ }^{1} \mathrm{H}$ magnetic resonance spectroscopy. Magn Reson Med, 2003, 49: 223-232

35 Govindaraju V, Young K, Maudsley A A. Proton NMR chemical shifts and coupling constants for brain metabolites. NMR Biomed, 2000, 13: 129-153

36 Kinoshita Y, Yokota A. Absolute concentrations of metabolites in human brain tumors using in vitro proton magnetic resonance spectroscopy. NMR Biomed, 1997, 10: 2-12

37 Cheng L L, Anthony D C, Comite A R, et al. Quantification of microheterogeneity in glioblastoma multiforme with ex vivo high-resolution magic-angle spinning (HRMAS) proton magnetic resonance spectroscopy. Neuro-oncology, 2000, 2: 87-95

38 Meyerand M E, Pipas J M, Mamourian A, et al. Classification of biopsy-confirmed brain tumors using single-voxel MR spectroscopy. AJNR Am J Neuroradiol, 1999, 20: 117-123

39 Koljenovic S, Choo-Smith L P, Bakker Schut T C, et al. Discriminating vital tumor from necrotic tissue in human glioblastoma tissue samples by Raman spectroscopy. Lab Invest, 2002, 82: 1265-1277

40 Fayed N, Davila J, Medrano J, et al. Malignancy assessment of brain tumours with magnetic resonance spectroscopy and dynamic susceptibility contrast MRI. Eur J Radiol, 2008, 67: 427-433

41 Roda J M, Pascual J M, Carceller F, et al. Nonhistological diagnosis of human cerebral tumors by ${ }^{1} \mathrm{H}$ magnetic resonance spectroscopy and amino acid analysis. Clin Cancer Res, 2000, 6: 3983-3993

42 Lehnhardt F G, Rohn G, Ernestus R I, et al. ${ }^{1} \mathrm{H}-$ and ${ }^{31} \mathrm{P}-\mathrm{MR}$ spectroscopy of primary and recurrent human brain tumors in vitro: malignancy-characteristic profiles of water soluble and lipophilic spectral components. NMR Biomed, 2001, 14: 307-317

43 Cheng L L, Chang I W, Louis D N, et al. Correlation of high-resolution magic angle spinning proton magnetic resonance spectroscopy with histopathology of intact human brain tumor specimens. Cancer Res, 1998, 58: 1825-1832

44 Wilson M, Davies N P, Brundler M A, et al. High resolution magic angle spinning ${ }^{1} \mathrm{H}$ NMR of childhood brain and nervous system tumours. Mol Cancer, 2009, 8: 6-16

45 Lukas L, Devos A, Suykens J A, et al. Brain tumor classification based on long echo proton MRS signals. Artif Intell Med, 2004, 31: 73-89

Open Access This article is distributed under the terms of the Creative Commons Attribution License which permits any use, distribution, and reproduction in any medium, provided the original author(s) and source are credited. 